\title{
Media optimization of Parietochloris incisa for arachidonic acid accumulation in an outdoor vertical tubular photobioreactor
}

\author{
Hazel Guevarra Tababa • Seishiro Hirabayashi • \\ Kazuyuki Inubushi
}

Received: 12 June 2011 /Revised and accepted: 5 August 2011 /Published online: 26 August 2011

(C) The Author(s) 2011. This article is published with open access at Springerlink.com

\begin{abstract}
The green alga Parietochloris incisa contains a significant amount of the nutritionally valuable polyunsaturated fatty acid and arachidonic acid (AA) and is being considered for mass cultivation for commercial AA production. This study was primarily aimed to define a practical medium formulation that can be used in commercial mass cultivation that will contribute to a substantial increase in the AA productivity of $P$. incisa with concomitant reduction of nutritional cost. The effect of nutrient limitation on growth and AA content of this microalga was explored in a batch culture in outdoor conditions using a vertical tubular photobioreactor. The study was conducted in two parts: the first was primarily focused on the effect of different nitrogen concentration on growth and AA content and the second part compares nitrogen deprivation, combination of nitrogen and phosphorus deprivation, and combined overall nutrient limitations at different levels of deprivation under low and high population densities. Since complete nitrogen deprivation hampers lipid and AA accumulation of $P$. incisa, thus, a critical value of nitrogen supply that will activate AA accumulation must be elucidated under specific growth conditions. Under the present experimental conditions, $0.5 \mathrm{~g}^{-1}$ sodium nitrate obtained a higher AA productivity and volumetric yield relative to the nitrogen-deprived culture corresponding to $36.32 \mathrm{mg} \mathrm{L}^{-1}$ day $^{-1}$ and $523.19 \mathrm{mg} \mathrm{L}^{-1}$. The combined nitrogen and phosphorus limitation seemed to enhance AA
\end{abstract}

H. G. Tababa $\cdot$ S. Hirabayashi

BioWing Co., Ltd, Minami-Oh-I 5-12-2,

Shinagawa-ku, Tokyo 140-0013, Japan

H. G. Tababa $\cdot$ K. Inubushi $(\bowtie)$

Graduate School of Horticulture, Chiba University,

Matsudo, Chiba 271-8510, Japan

e-mail: inubushi@faculty.chiba-u.jp productivity better than nitrogen deprivation alone. The effect of overall nutrient limitation indicates that acute nutrient deficiency can trigger rapid lipid and AA syntheses. The effect of light as a consequence of culture cell density was also discussed. This study therefore shows that the nutrient cost can be greatly reduced by adjusting the nutrient levels and culture density to induce AA accumulation in P. incisa.

Keywords Arachidonic acid - Commercial mass cultivation - Combined nitrogen and phosphorus limitation. Nitrogen deprivation - Vertical tubular photobioreactor. Chlorophyta

\section{Introduction}

Many photoautotrophic microalgae have the capacity to produce appreciable amounts of oil and are considered as potential candidates for commercial mass cultivation to produce valuable long-chain polyunsaturated fatty acids (LCPUFA; Shifrin and Chisholm 1981; Thompson 1996; Molina-Grima et al. 1999). Nutritionally important LCPUFA include eicosapentaenoic acid (20:5 $\omega 3$, EPA), docosahexaenoic acid (22:6w3, DHA), and arachidonic acid (20:4w6, AA). EPA and DHA are found abundant in microalgae. Phaeodactylum tricornutum (Yongmanitchai and Ward 1992), Monodus subterraneous ( $\mathrm{Hu}$ et al. 1997), Porphyridium cruentum (Cohen 1999), and Nannochloropsis sp. (Seto et al. 1984) were well studied due to their potential as source of EPA and Schizochytrium (Wu et al. 2005), Crypthecodinium cohnii (Jiang et al. 1999), and Chroomonas salina (Henderson and Mackinlay 1992) for DHA. Arachidonic acid, however, is notably rare, and if found present in marine species, it amounts to a few percent of total fatty acids (Thompson 1996). A marine microalgal 
species, $P$. cruentum, has been reported to produce AA in appreciable amount under specific growth conditions (Cohen 1990). However, the freshwater green microalga, Parietochloris incisa (Trebuxiophyceae, Chlorophyta), isolated from the snowy slope of Mt. Tateyama, Japan (Watanabe et al. 1996), is exceptionally capable of accumulating high amounts of AA. P. incisa is recognized as the richest vegetal sources of AA (Khozin-Goldberg et al. 2002) and is being considered for mass cultivation as a vegetal source of AA.

Algae alter their fatty acid content and composition in response to environmental conditions (Thompson 1996). Under optimal growth conditions, microalgae synthesize fatty acids for the formation of membrane lipids with polyunsaturated fatty acids as major constituents (Erwin 1973). In adverse conditions for growth, microalgae generally accumulate neutral lipids, mainly as triacylglycerol (TAG; Shifrin and Chisholm 1981). TAG functions mainly as a storage material, in contrast to the membrane lipids that perform a structural role. This phenomenon is due to the shift in lipid metabolism from membrane lipid synthesis to the storage of neutral lipids. TAGs are composed primarily of $\mathrm{C} 14-\mathrm{C} 18$ fatty acids that are saturated or mono-unsaturated (Cohen 1986; Harwood 1998; Roessler 1990). Among the factors that are known to affect TAG synthesis in microalgal cells are nutrient starvation, light intensity, temperature, medium $\mathrm{pH}$, and salinity (Hu et al. 2008).

Nutrient stress is the most commonly used method to induce lipid accumulation. Specific nutrients such as nitrogen, phosphorus, sulfur, and silica are usually eliminated from the growth medium, individually or in combination, to effect lipid accumulation. Nitrogen limitation is the most extensively studied nutrient and is considered as the most critical in affecting lipid metabolism in algae. Many microalgal species exhibit a general trend of accumulating lipids, mostly TAG, in response to nitrogen deficiency (Shifrin and Chisholm 1981; Roessler 1990; Thompson 1996; Guschina and Harwood 2006). Phosphate limitation has been reported to promote lipid synthesis (Reitan et al. 1994) and results in the increase in lipid content of Monodus subterraneus (Eustigmatophyceae; Khozin-Goldberg and Cohen 2006), whereas Chorella sp. (Otsuka 1961) and Chlamydomonas reinhardtii (Sato et al. 2000) was also shown to enhance lipid content under sulfur deprivation.

One factor that limits the commercial realization of a potential microalga for mass cultivation is the prohibitive cost of photoautotrophic mass cultivation. Research dealing on reducing cost in production system is an integral part in developing an algal mass cultivation technology. The use of wastewater as feed has been widely recommended; however, the applicability of using wastewater in microalgal cultivation intended for human consumption may not be readily acceptable. A practical approach to significantly reduce the operating cost of microalgal cultivation would be the manipulation of nutrient feed at the minimum level to attain the maximum productivity of the desired product without a significant reduction in biomass productivity. Nutrient is one of the factors contributing to the prohibitive cost of photoautotrophic microalgal mass cultivation. Therefore, this study aims to elucidate the optimal nitrogen concentration for total fatty acid (TFA) and AA accumulation and to formulate a practical medium composition at a reduced cost without sacrificing the productivity of AA accumulation in an outdoor mass cultivation of $P$. incisa.

\section{Materials and methods}

Organism and culture maintenance

Cultures of $P$. incisa were cultivated under $22 \pm 0.2^{\circ} \mathrm{C}$ in a 300-mL flask containing $150 \mathrm{~mL}$ Bold Basal Medium (BBM) composed of (per liter) $1.5 \mathrm{~g} \mathrm{NaNO}_{3}, 0.075 \mathrm{~g} \mathrm{~K}_{2} \mathrm{HPO}_{4}$, $0.175 \mathrm{~g} \mathrm{KH}_{2} \mathrm{PO}_{4}, 0.075 \mathrm{~g} \mathrm{MgSO}_{4} \cdot 7 \mathrm{H}_{2} \mathrm{O}, 0.084 \mathrm{~g}$ $\mathrm{CaCl}_{2} \cdot 2 \mathrm{H}_{2} \mathrm{O}, 0.00498 \mathrm{~g} \mathrm{FeSO}_{2} \cdot 7 \mathrm{H}_{2} \mathrm{O}, 0.05$ g EDTA $2 \mathrm{Na}$ salt, $0.025 \mathrm{~g} \mathrm{NaCl}, 0.031 \mathrm{~g} \mathrm{KOH}, 11.42 \mu \mathrm{g} \mathrm{H}_{3} \mathrm{BO}_{3}, 1.44 \mu \mathrm{g}$ $\mathrm{MnCl}_{2} \cdot 4 \mathrm{H}_{2} \mathrm{O}, 8.82 \mu \mathrm{g} \mathrm{ZnSO}{ }_{4} \cdot 7 \mathrm{H}_{2} \mathrm{O}, 1.57 \mu \mathrm{g} \mathrm{CuSO}_{4} \cdot 5 \mathrm{H}_{2} \mathrm{O}$, $0.049 \mu \mathrm{g} \mathrm{Co}\left(\mathrm{NO}_{3}\right)_{2} \cdot 6 \mathrm{H}_{2} \mathrm{O}$, and $0.71 \mu \mathrm{g} \mathrm{MoO}_{3}$.

Inocula for outdoor experiments were cultured in 5-L Erlenmeyer flasks containing $4 \mathrm{~L}$ medium and mixed by air containing $1 \% \mathrm{CO}_{2}(v / v)$ at a flow rate of $0.2 \mathrm{~L} \mathrm{~min}^{-1}$. Cultures were maintained by changing medium at 2 weeks interval.

Vertical tubular photobioreactors (VTPR) with $5-\mathrm{cm}$ diameter, 2-m height, and a working volume of $4 \mathrm{~L}$ were used for outdoor cultivation. Laboratory-grown inoculum was transferred to the VTPR at least 4 days prior to the start of the experiment. Mixing and $\mathrm{CO}_{2}$ were provided by enriched air with $1 \% \mathrm{CO}_{2}(v / v)$ at the rate of $1 \mathrm{~L} \mathrm{~min}^{-1}$ through perforations at the bottom of the reactor. The rate of gas flow was controlled by a flow meter. The temperature was controlled by water mist, and temperature did not exceed $30^{\circ} \mathrm{C}$.

These experiments were conducted in November and February-March in Tokyo, Japan. The average daily solar irradiations $\left(\mathrm{kWh} \mathrm{m}^{-2} \mathrm{day}^{-1}\right.$ ) per month were 2.77 and 3.72 4.17 for November and February-March, respectively (source: Atmospheric Science Data Center, NASA Surface meteorology and Solar Energy; http://eosweb.larc.nasa.gov/).

For nitrogen concentration evaluation, sodium nitrate concentration was varied from 0 to $1.5 \mathrm{~g} \mathrm{~L}^{-1}$. To investigate the overall nutrient deficiency, BBM with $0.5 \mathrm{~g} \mathrm{~L}^{-1}$ sodium nitrate was used as the base medium. The deficient media were prepared to give nutrient concentrations of $75 \%, 50 \%$, $25 \%, 10 \%$, and $0 \%$ (tap water only) of the base medium and base medium without nitrogen (BBM-N) and the base medium without nitrogen and without phosphorous (BBMN-P). This experiment was carried out under two initial 
population densities of 1.8 and $>5 \mathrm{~g} \mathrm{~L}^{-1}$. Algal cells were washed twice before being suspended in treatment medium.

\section{Growth measurements}

For dry biomass determination, $10 \mathrm{~mL}$ of culture was filtered on tared, pre-dried GF/C Whatman filters. Filtered biomass was washed with $10 \mathrm{~mL}$ of acidified distilled water $(\mathrm{pH} 4)$ to remove inorganic salts and dried at $105^{\circ} \mathrm{C}$ for $3 \mathrm{~h}$. Dried samples were allowed to cool to room temperature in a desiccator over silica gel before being weighed.

For chlorophyll determination, $2 \mathrm{~mL}$ of the culture was centrifuged at $2,000 \times g$ for $5 \mathrm{~min}$. Pigments were extracted from algal pellets with DMSO in $70^{\circ} \mathrm{C}$ water bath for $20 \mathrm{~min}$. The extracts were mixed, centrifuged at $2,000 \times \mathrm{g}$ for $5 \mathrm{~min}$, and measured spectrophotometrically based on Wellburn (1994).

For fatty acid analysis, $50 \mathrm{~mL}$ of the culture was centrifuged at $2,000 \times \mathrm{g}$ for $5 \mathrm{~min}$ and the algal pellets were immediately frozen and freeze-dried. Total lipid of the dry biomass was extracted following the method of Bligh and Dyer (1959). Lipid extracts were transmethylated with methanol-acetyl chloride according to Cohen et al. (1993); heptadecanoic acid (C17) was added as an internal standard. Fatty acid methyl esters were identified by cochromatography with authentic standards (Supelco, USA) and by calculation of the equivalent chain length. The methyl esters were analyzed with a Shimadzu GC-8A using Supelco 2560 capillary column $(100 \mathrm{~m} \times 0.25 \mathrm{~mm})$ at $150^{\circ} \mathrm{C}$ (FID, injector and flame ionization detector temperature $250^{\circ} \mathrm{C}$, split ratio $1: 100$ ). The rate of temperature increase was $3^{\circ} \mathrm{C} \mathrm{min}^{-1}$. Fatty acid content was determined by comparing each peak area with that of the internal standard and corrected accordingly.

\section{Results}

To understand the effect of nitrogen concentration on the growth and AA accumulation of $P$. incisa under outdoor conditions exposed to full sunlight, sodium nitrate was varied from 0 to $1.5 \mathrm{~g} \mathrm{~L}^{-1}$. The change in biomass productivity shows that the final biomass did not differ in all treatments at the end of the cultivation period (Fig. 1), attaining biomass of $5.3 \mathrm{~g} \mathrm{~L}^{-1}$ for nitrogen-supplied cultures and $5.0 \mathrm{~g} \mathrm{~L}^{-1}$ for nitrogen-deficient (BBM-N) at day 14. Chlorophyll gradually declines in all treatments (Fig. 2), with the reduction in chlorophyll biomass content more prominent in the culture provided with lower nitrogen concentrations. In all of the treatments, both TFA and AA increased through the cultivation period. Fatty acid accretion was noticeably faster in BBM-N in comparison with cultures supplied with nitrogen (Fig. 3a). P. incisa grown in

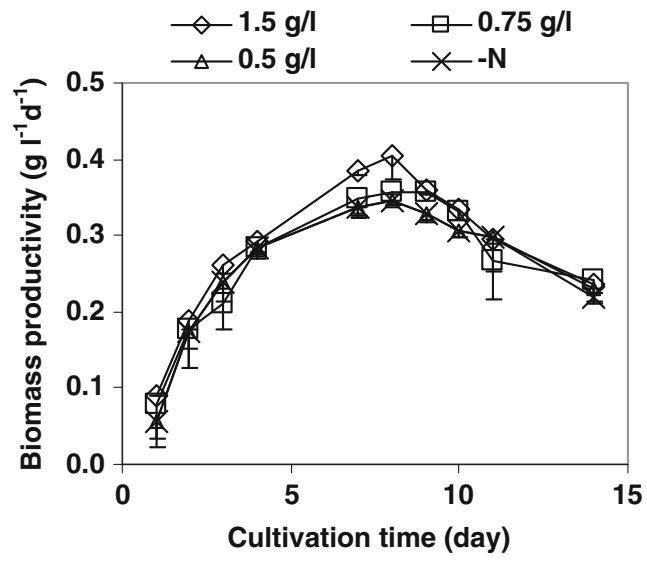

Fig. 1 Biomass productivity of P. incisa cultivated outdoor under different nitrogen concentrations

$0.5 \mathrm{~g} \mathrm{~L}^{-1} \mathrm{NaNO}_{3}$ and BBM-N both attained TFA content of $20 \%$ of dry biomass, whereas cultures supplied with 1.5 and $0.75 \mathrm{~g} \mathrm{~L}^{-1} \mathrm{NaNO}_{3}$ reached $14.1 \%$ and $16.7 \%$, respectively (Fig. 3a). The proportion of AA increased at the same rate up to 9 days of incubation for $0.75 \mathrm{~g} \mathrm{~L}^{-1}$ $\mathrm{NaNO}_{3}$ to BBM-N; however, the rate diminished thereafter for BBM-N and $0.75 \mathrm{~g} \mathrm{~L}^{-1} \mathrm{NaNO}_{3}$, while cultures in $0.5 \mathrm{~g} \mathrm{~L}^{-1} \mathrm{NaNO}_{3}$ continued to increase, accounting for the higher AA proportion (Fig. 3b). The lowest proportion of AA in TFA was obtained in N-replete culture with $25.9 \%$, while cultures supplied with 0.75 and $0.5 \mathrm{~g} \mathrm{~L}^{-1} \mathrm{NaNO}_{3}$ and BBM-N attained AA proportions of $41.0 \%, 47.4 \%$, and $39.7 \%$, respectively (Fig. 3b). The final AA contents after 14 days of cultivation were $3.7 \%, 6.8 \%, 9.9 \%$, and $6.3 \%$ of dry biomass (Fig. 3c) corresponding to volumetric AA contents of 203.1, 361.0, 523.2, and $416.5 \mathrm{mg} \mathrm{L}^{-1}$ and AA productivity of $13.4,25.0,36.3$, and $28.5 \mathrm{mg} \mathrm{L}^{-1}$ day $^{-1}$ in cultures supplied with $1.5,0.75$, and $0.5 \mathrm{~g} \mathrm{~L}^{-1} \mathrm{NaNO}_{3}$ and BBM-N, respectively (Fig. 4).

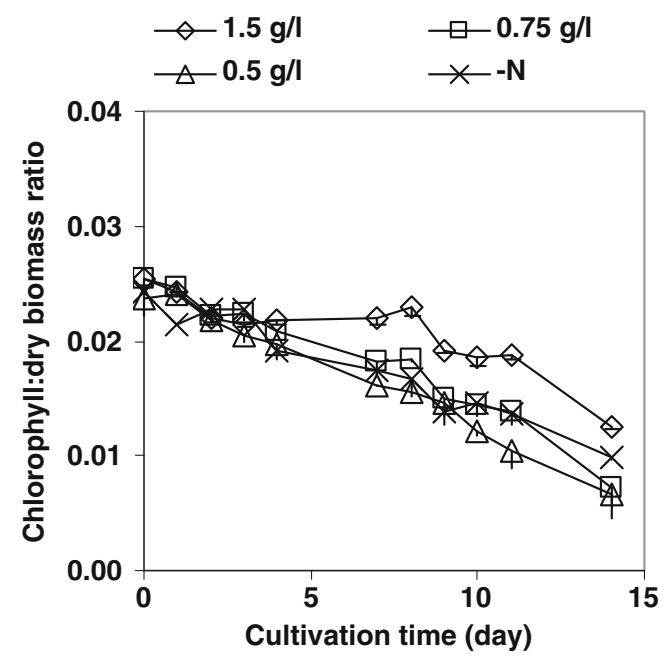

Fig. 2 Chlorophyll content of $P$. incisa cultivated outdoor under different nitrogen concentrations 

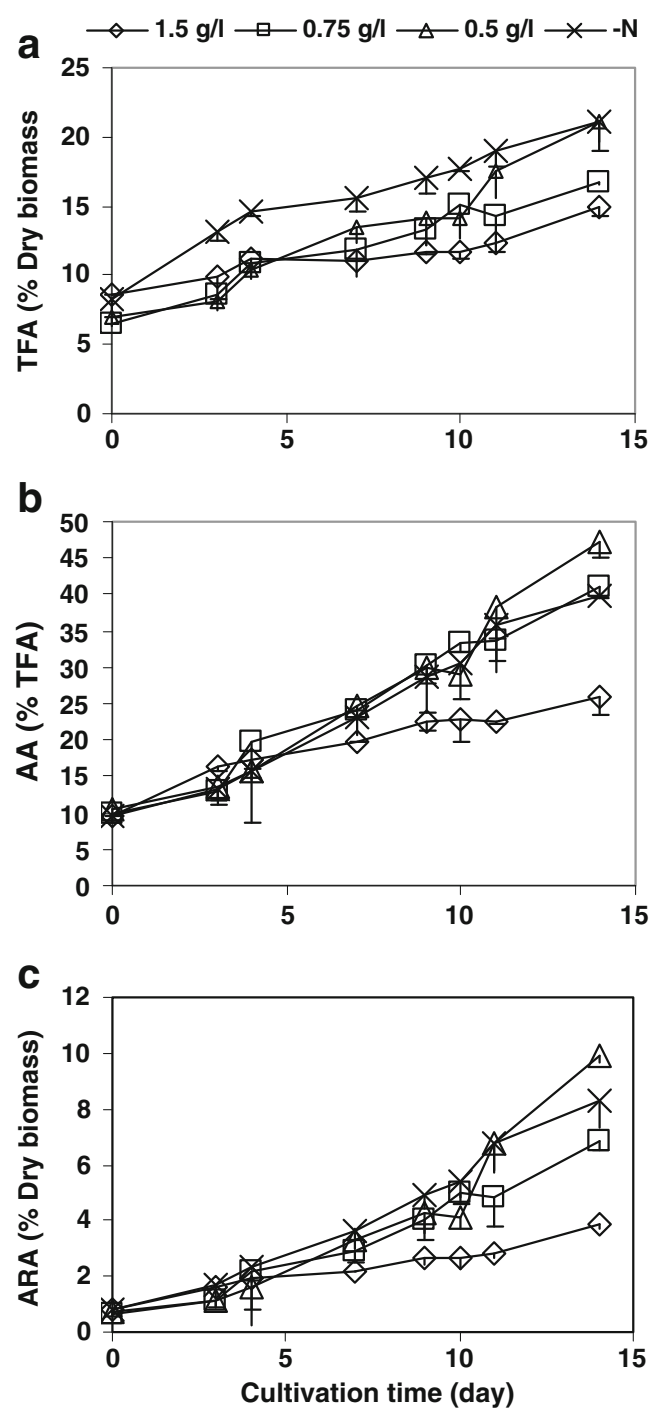

Fig. 3 Changes in total fatty acid (TFA) content (a), arachidonic acid $(A A)$ proportion in TFA (b), and AA content (c) of $P$. incisa in different sodium nitrate concentrations

The effect of combined deficiencies of all the nutrients supplied in the base medium as well as nitrogen deficiency and combined nitrogen and phosphorous deficiency on the growth and fatty acid and AA content of $P$. incisa was explored. The base medium used for the second test for AA accumulation media optimization was BBM with $0.5 \mathrm{~g} \mathrm{NaNO}_{3} \mathrm{~L}^{-1}(0.5 \mathrm{~N} \mathrm{BBM})$, which also served as the control. The deficient media contained $75 \%, 50 \%, 25 \%$, $10 \%$, and $0 \%$ (tap water, TW) of the base medium, nitrogen-deficient base medium (BBM-N), and nitrogenand phosphorous-deficient base medium (BBM-N-P). The different media formulations were evaluated under low density (LD, $\left.2 \mathrm{~g} \mathrm{~L}^{-1}\right)$ and high density $\left(\mathrm{HD},>5 \mathrm{~g} \mathrm{~L}^{-1}\right)$. The cultures of LD and HD exhibited a different growth profile where LD cultures regardless of media formulation exhibited a normal growth curve (Fig. 5a), whereas HD
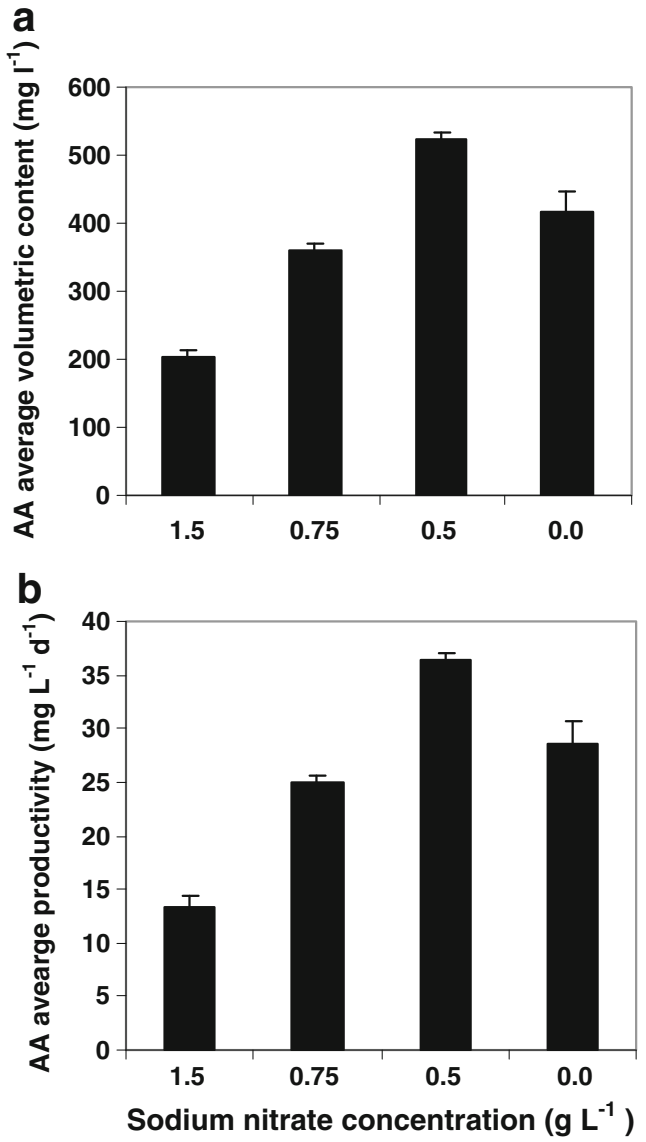

Fig. 4 Arachidonic acid volumetric content (a) and productivity (b) of $P$. incisa grown in different sodium nitrate concentrations after 14 days of cultivation

exhibited a relatively linear growth. In LD cultures, $0.5 \mathrm{~N}$ $\mathrm{BBM}$ and TW reached maximum productivity at 8 days; the other treatments attained maximum productivity at 12 days, at which point the productivity declined. In HD culture, biomass productivity increased up to 7 days of cultivation and generally remained constant up to 14 days. The productivity is positively correlated to the amount of nutrient in the media, with tap water attaining the lowest biomass productivity (Fig. 5).

Increments in fatty acids, AA proportion, and AA biomass of the content of HD cultures are shown in Fig. 6. Cultures in 10\% BBM,-N-P BBM, and TW consistently exhibited higher TFA, AA proportion, and AA biomass content relative to the other treatments, with the AA content of biomass in these media all reaching $+10 \%$ (Fig. 6c). The fatty acid content of BBM-N increased up to 10 days, at which point it gradually declined, whereas $0.5 \mathrm{~N} \mathrm{BBM}$ and 75,50 , and 25\% BBM continue to increase in TFA (Fig. 6a). This resulted in BBM-N obtaining the lowest TFA content. The proportion of AA declined in $0.5 \mathrm{~N}$ BBM, $75 \%, 50 \%$, $25 \%$ BBM, and BBM-N up to 7 days. The rate of AA accumulation of $0.5 \mathrm{~N}$ BBM, 75, 50, and 25\% BBM 
Fig. 5 Changes in biomass $(\mathbf{a}, \mathbf{b})$ and productivity $(\mathbf{c}, \mathbf{d})$ of $P$. incisa grown under varying nutrient deficiencies at low initial cell density $(\mathbf{a}, \mathbf{c})$ and high initial population density $(\mathbf{b}, \mathbf{d})$
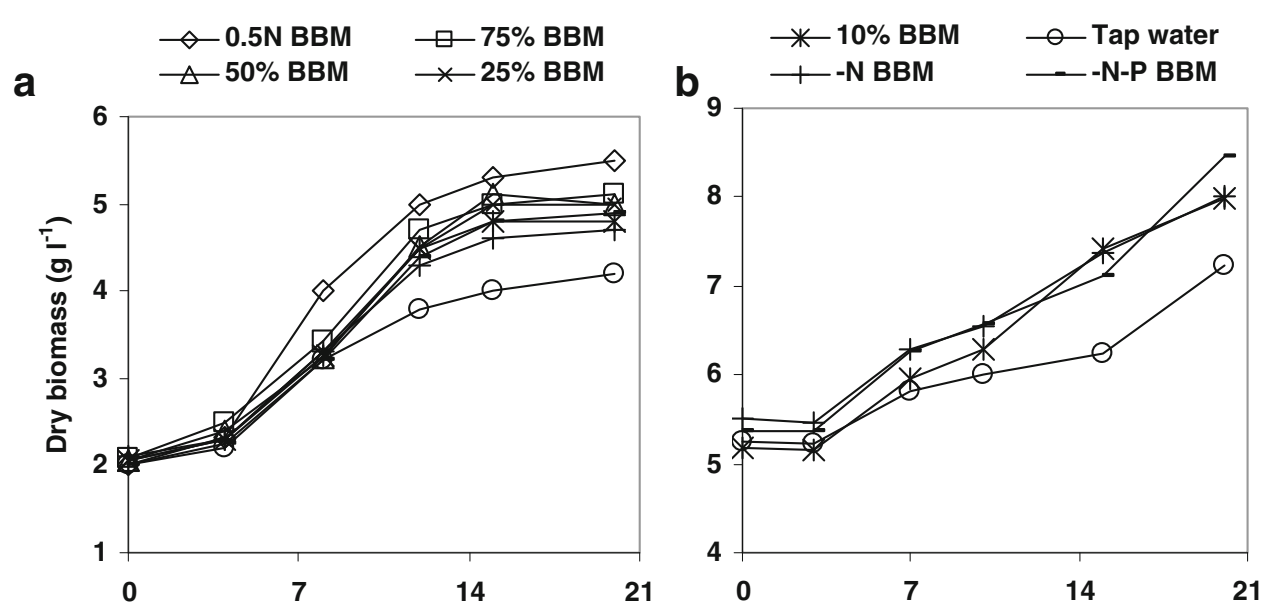

C

C

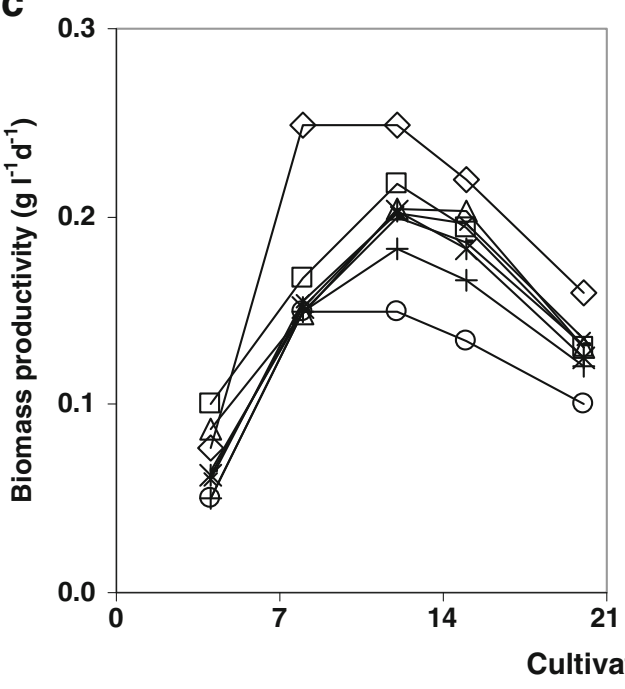

d

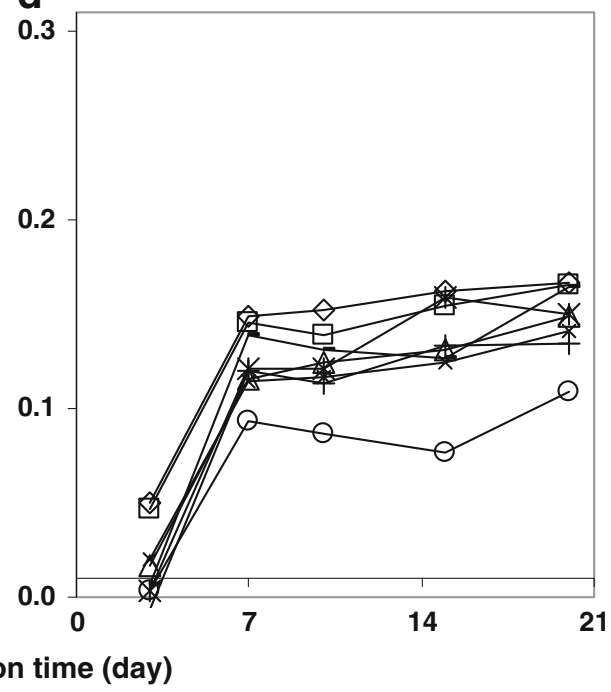

gradually increased up to 10 days and peaked thereafter. In contrast, BBM-N exhibited a strong increase in AA proportion up to 10 days, and the rate of accumulation gradually tapered off up to 20 days (Fig. 6b).

In both $\mathrm{LD}$ and $\mathrm{HD}$ cultures, the combined nutrient deficiencies enhanced TFA content and AA proportion in $P$. incisa. The TFA content of biomass obtained for LD and HD were similar; however, a higher proportion of AA was observed in HD cultures over LD cultures, consequently resulting in higher AA biomass content (Fig. 7a, c) and volumetric culture content in HD cultures (Fig. 7b, d).

Among LD cultures, 10\% BBM, TW, -N-P BBM, 25\% $\mathrm{BBM}$, and 50\% BBM (in decreasing order) exhibited a higher AA biomass content relative to the control $(0.5 \mathrm{~N}$ BBM; Fig. 7a). However, though TW reached higher AA biomass content over the other media tested, TW obtained the lowest volumetric content (Fig. 7b) due to the low culture biomass attained at the end of the cultivation period. In $\mathrm{HD}$ cultures, $10 \% \mathrm{BBM}$, TW, and $-\mathrm{N}-\mathrm{P}$ BBM reached a higher AA biomass content and AA volumetric culture content over the other treatments (Fig. 7c, d).

\section{Discussion}

One of the main factors that hinder the economic feasibility of a microalgal mass cultivation for valuable polyunsaturated fatty acids (PUFA) production is the prohibitive cost in initial capital investment as well as the nutritional requirements and downstream processing. Fundamental parameters that affect the economic feasibility of algal mass cultivation are biomass productivity, cell content of PUFA, and overall PUFA productivity since these essentially affect the costs of cultivation and downstream processing for extraction of purification of the valuable PUFA. Achieving the highest biomass productivity with the highest content of the PUFA is ideal; however, substantial PUFA production in algal cells usually occurs under stress conditions where biomass productivity is typically low. One of the factors which greatly affect biomass productivity and secondary metabolite accumulation that can be easily manipulated is nutrient. Nutrient cost demands a considerable portion in the production cost. The use of nutrient-rich wastewaters or inexpensive agricultural fertilizers has been 

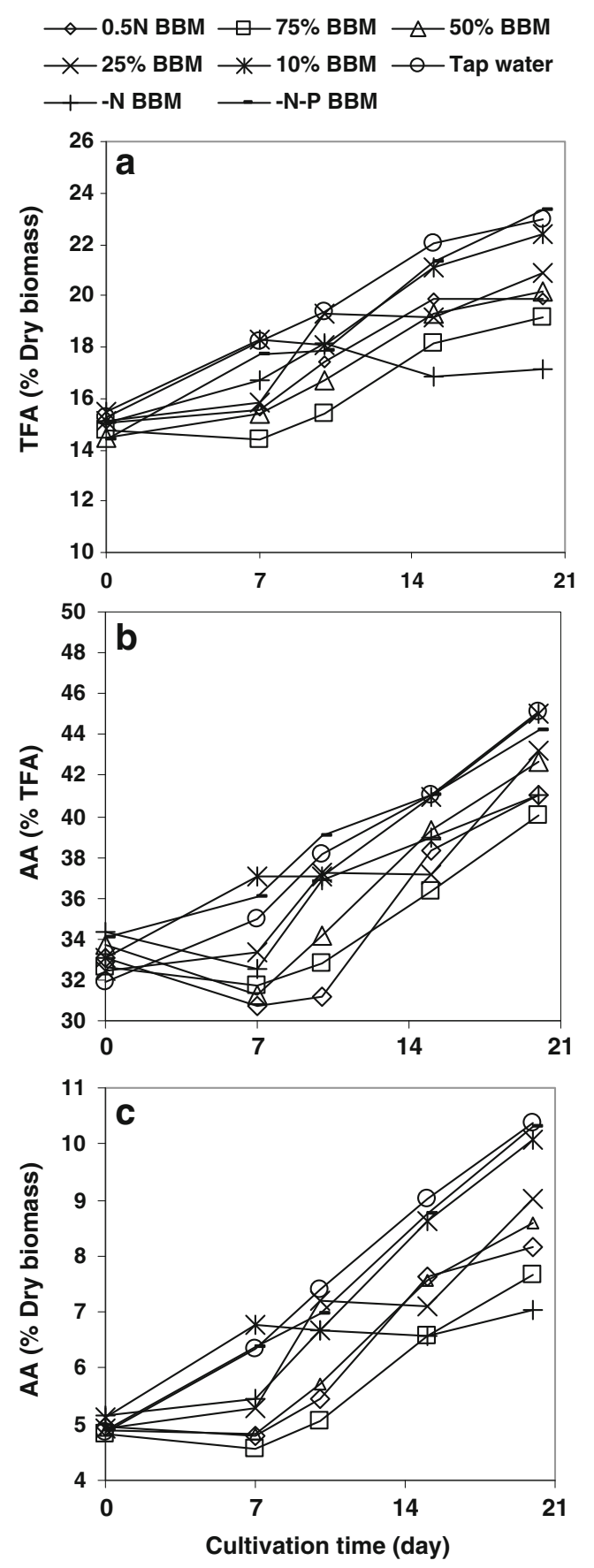

Fig. 6 Changes in total fatty acid (TFA) content (a), arachidonic acid $(A A)$ proportion in TFA (b) and AA content (c) of $P$. incisa grown with varying nutrient deficiencies at high initial population density

recommended; however, these nutrient sources may not be appropriate for microalgae production intended for human consumption. A practical approach would be the manipulation of nutrient supply to modulate growth and PUFA accumulation in the algal cell. A microalgal mass cultivation for valuable PUFA production aims for high yield of PUFA as a function of biomass productivity and PUFA content in the biomass.
In general, microalgae accumulate lipid under stress conditions which are basically unfavorable to growth. Several studies aimed at achieving maximal cell contents and overall productivities of PUFA in microalgae have focused primarily on nutrient stress (Otsuka 1961; Reitan et al. 1994; Sato et al. 2000; Khozin-Goldberg and Cohen 2006). Nitrogen starvation has been reported to enhance fatty acid content in chlorophycean algae (Shifrin and Chisholm 1981). Generally, in microalgae, nitrogen starvation will result in the accumulation of large quantities of TAG (Packer et al. 2011), but is accompanied by a steep decline in both the proportion and content of PUFA (Cohen 1986). P. incisa seemed to be the exception since it has the ability to accumulate AA-rich TAG, as reported by Bigogno et al. (2002a, b). Extensive studies on the effect of nitrogen deprivation on the AA accumulation of $P$. incisa have shown that nitrogen deprivation greatly enhanced AA production (Khozin-Goldberg et al. 2002; Cheng-Wu et al. 2002; Merzlyak et al. 2007; Solovchenko et al. 2008). Since $P$. incisa is being considered for algal mass cultivation for commercial AA production, the response of $P$. incisa to different levels of nitrogen limitations was evaluated in an outdoor condition. Studies on the effect of different levels of nitrogen on the accumulation of AA in these microalgae are lacking since most of the report available compares only nitrogen-replete and nitrogen-deprived cultures.

The results showed no differences in the biomass productivity of cultures grown in different concentrations of nitrogen from $1.5 \mathrm{~g} \mathrm{NaNO}_{3} \mathrm{~g} \mathrm{~L}^{-1}$ to $\mathrm{N}$-deficient levels at 14 days of cultivation period. For N-replete cultures, the biomass productivity was higher than in N-limited cultures at 8 days. The subsequent decline of productivity in N-replete cultures was due to light limitation when the culture reached a high population density rather than nitrogen depletion. The increase in biomass at nitrogen limited cultures is partly attributed to the accumulation of lipids rather than due to cell growth. This is supported by the defined increase in the TFA and AA contents of the nitrogen-limited cultures. Nitrogen limitation reduces cell growth, but photosynthetic carbon fixation continues until a certain critical level is reached. Upregulation of lipid synthesis has been proposed to be a default pathway by which energy can be spent during stressed conditions where cell growth is reduced, helping to maintain a safe turnover rate of the ATP and reductant pools sustained by the light reactions (Hu et al. 2008).

Regardless of nitrogen concentration in the medium, the AA proportion, TFA and AA contents in biomass increased through time. This shows the effect of culture aging which enhances the proportion of AA and TFA contents, as reported by Khozin-Goldberg et al. (2002). The fatty acid content and the proportion of AA were shown to be negatively correlated to the concentration of nitrogen in the culture medium. The effect of nitrogen deficiency was 

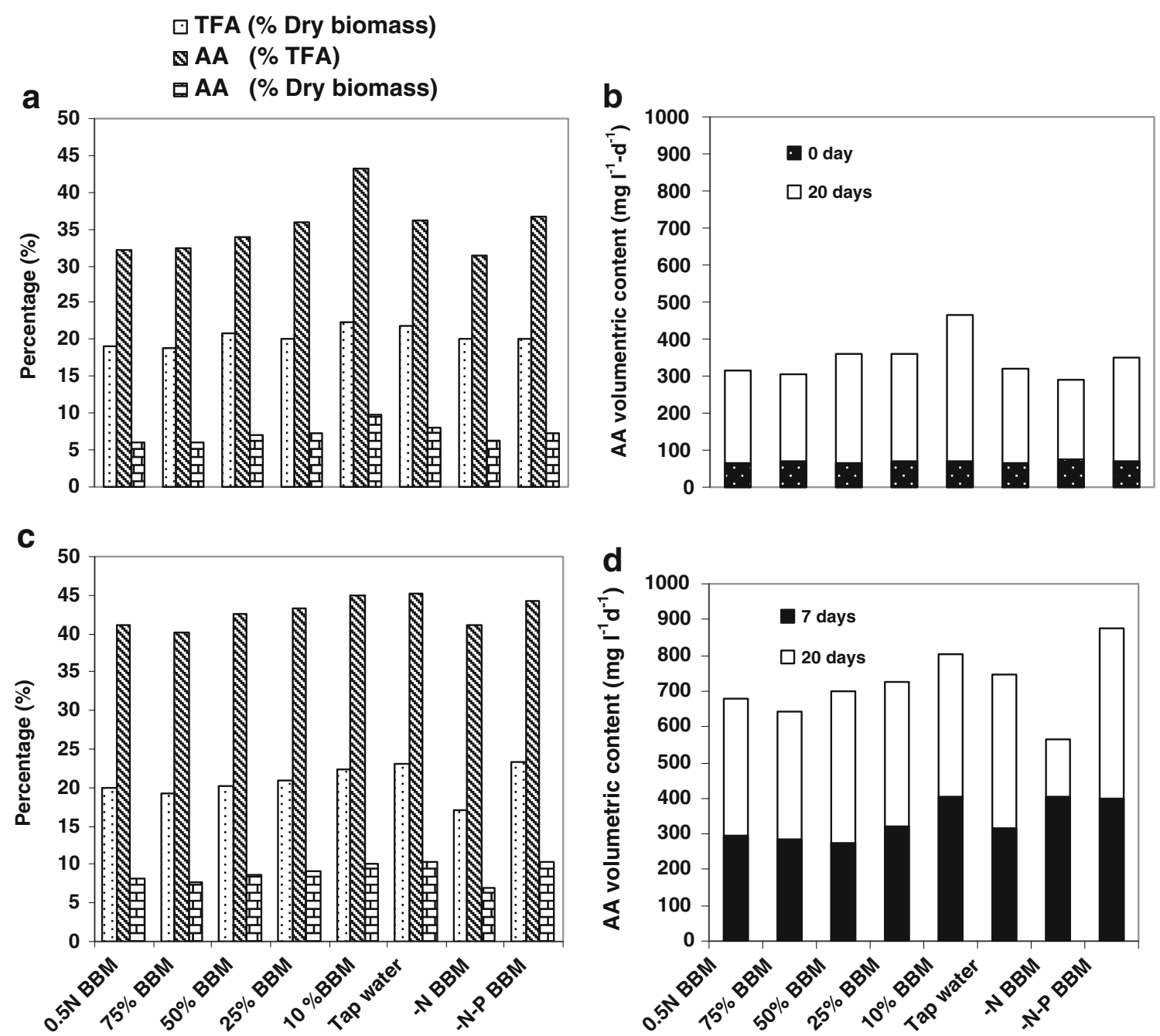

Fig. 7 Total fatty acid (TFA) content, arachidonic acid $(A A)$ proportion in TFA and AA content (a, c), and arachidonic acid volumetric content (b, c) of $P$. incisa under varying nutrient deficiencies at low initial population density $(\mathbf{a}, \mathbf{c})$ and high initial population density $(\mathbf{b}, \mathbf{d})$

more prominent in fatty acid accumulation since TFA distinctly increased at a faster rate in the nitrogendeficient medium (BBM-N). However, acute nitrogen deficiency seemed to inhibit the synthesis of AA at the later stage of incubation where a marked decline in the rate of AA accumulation was observed after 10 days. It can be hypothesized that under severe nitrogen limitation, the photosynthetic activity was significantly slowed toward the end of the cultivation period, resulting in an overall decrease in TFA and AA proportion in TFA. In microalgae, nitrogen limitation affects photosynthesis by reducing the efficiency of energy collection due to the loss of Chl $a$ and the increase in non-photochemically active carotenoid pigments (Herzig and Falkowski 1989; Geider et al. 1993) and directly affects photochemical energy conversion because of decrease in protein synthesis that appear to affect chloroplastic proteins (and thus the proteins of PSI and PSII reaction centers) more strongly than cytoplasmic proteins (Rhiel et al. 1986; Kolber et al. 1988; Falkowski et al. 1989; Geider et al. 1993). In this experiment, a more prominent reduction in chlorophyll content was observed in nitrogen-limited cultures. The decline in chlorophyll is a general response of microalgae to nitrogen deficiency due to the inhibition of protein synthesis. Lipid synthesis is a high-energy-consuming process where, on a per-mass basis, neutral lipid synthesis requires twice the reducing energy (NADPH) than that of carbohydrate or protein synthesis (Hu et al. 2008). Thus, in these experiments, with inadequate photosynthetic activities brought about by severe nitrogen limitation, the cells may not have enough fixed carbon as well as enough reducing energy for lipid synthesis toward the end of the cultivation period in the BBM-N cultures, resulting in an overall decline in fatty acid and AA biosynthesis so that the maximum potential of the alga for AA production may not be achieved. The final AA productivity and AA volumetric culture content was higher at $0.5 \mathrm{~g} \mathrm{~L}^{-1} \mathrm{NaNO}_{3}$ than in the BBM-N cultures. This suggests that the complete removal of nitrogen from the medium 
could hamper the potential AA productivity of $P$. incisa and that the critical nitrogen concentration in the growth medium that will induce and sustain fatty acid and AA accumulation throughout the culture cycle must be identified. This phenomenon must be considered in developing a mass cultivation system of $P$. incisa for AA production.

In the combined nutrient deficiency evaluation, the different growth profile observed in LD and HD cultures was due to light availability in the culture as affected by population density. The low biomass productivities attained in HD cultures was attributed to light limitation. Solovchenko et al. (2008) reported that under light-limiting conditions, $P$. incisa exhibited a slow linear growth regardless of the presence of nitrogen. In both LD and HD, it is shown that severe nutrient limitation reduces the biomass productivity of the alga, and the decline in productivity positively correlated with the nutrient concentrations in the medium. Thus, tap water-grown cultures obtained the lowest biomass productivity in both LD and HD cultures.

The changes in the fatty acid and AA contents of HD cultures, as shown in Fig. 6, demonstrate the effect of different nutrient limitation in $P$. incisa. Cultures grown in BBM-N-P, $10 \%$ BBM, and tap water enhanced both TFA and AA proportions from the early stage of cultivation, while a 7-day lag was observed in the other treatments. TFA and AA accumulation was sustained in all cultures up to the end of the cultivation period, except for BBM-N where TFA and AA accumulation declined towards the end of the cultivation period. A different pattern of TFA and AA accumulation was observed between the first experiment (different nitrogen concentration) and the second experiment (nutrient limitation). This can be attributed to the difference in light received by the culture since the initial density was different in the two experiments where a low initial population density $\left(1.8 \mathrm{~g} \mathrm{~L}^{-1}\right)$ was used in the first experiment and a high density $\left(>5 \mathrm{~g} \mathrm{~L}^{-1}\right)$ in the second experiment. Unfortunately, the TFA and AA accumulation pattern was not monitored in the LD cultures of the second experiment. However, in both experiments, the data demonstrated that the rate of AA and TFA accumulation was not sustained throughout the cultivation period in BBM$\mathrm{N}$, and the rate of AA accumulation in biomass declined towards the end of cultivation period, resulting in low final AA biomass content.

The effect of light as a consequence of the difference in population density was also evident in the final AA proportion and AA biomass content achieved by LD and HD cultures. TFA values obtained were relatively similar between the same treatments of LD and HD cultures; however, cultures of the same media formulations exhibited higher AA proportions and AA biomass content in lightlimited HD cultures than in LD cultures. This result agrees with other reports that high light facilitates rapid growth but low AA content, and light intensities limiting for growth favor high AA biomass content in P. incisa (Cheng-Wu et al. 2002; Solovchenko et al. 2008).

Under nutrient limitation, total fatty acid and the proportion of AA increased, but cellular growth declined. Combined nitrogen and phosphorus deficiency exhibited a more pronounced effect on the AA content of biomass and the proportion of AA than nitrogen deficiency alone. Furthermore, it was rather surprising that $10 \% \mathrm{BBM}$, BBM-N-P, and TW reached an appreciable higher final AA content as compared with the other media formulations used. The better performance of 10\% BBM, BBM-N-P, and TW may be due to the synergistic effect of combined nutrient stress. In Haematococcus pluvialis, accumulation of astaxanthin was greatly enhanced by the combined stress of high light, salt, and iron than when stress was applied separately (Steinbrenner and Linden 2001). The combination of high salinity with high light intensity and high temperature also has been reported to increase cellular contents of $\beta$-carotene in Dunaliella (Borowitzka and Borowitzka 1988). This shows that a synergistic effect of different stress conditions if applied may also increase further the AA productivity in $P$. incisa.

The higher AA content in the biomass of nutrient-limited cultures grown at $\mathrm{HD}$, i.e., $10 \% \mathrm{BBM}$ and tap water, compensated for the biomass productivity loss. The tradeoff between the reduction in biomass productivity and the increase in the arachidonic acid content must be clearly balanced in algal mass cultivation. These results demonstrate that manipulation of light environment through adjustment of the population density and provision of optimal nutrient levels to modulate biomass productivity and AA accumulation can be an effective tool to increase AA productivity at a reduced cost that will bring the $P$. incisa mass cultivation technology for AA production closer to commercial realization.

Acknowledgments This research was supported by T. Akiyama and Co. and Naigai Chemical Products Co., Ltd., Tokyo, Japan.

Open Access This article is distributed under the terms of the Creative Commons Attribution Noncommercial License which permits any noncommercial use, distribution, and reproduction in any medium, provided the original author(s) and source are credited.

\section{References}

Bigogno C, Khozin-Goldberg I, Adlerstein D, Cohen Z (2002a) Biosynthesis of arachidonic acid in the oleaginous microalga Parietochloris incisa (Chloropyceae): radiolabeling studies. Lipids 37:209-216

Bigogno C, Khozin-Goldberg I, Boussiba S, Vonshak A, Cohen Z (2002b) Lipid and fatty acid composition of the green oleaginous alga Parietochloris incisa, the richest plant source of arachidonic acid. Phytochem 60:497-503 
Bligh EG, Dyer WJ (1959) A rapid method of total lipid extraction and purification. Can J Biochem Physiol 37(8):911-917

Borowitzka MA, Borowitzka LJ (1988) Dunaliella. In: Borowitzka MA, Borowitzka LJ (eds) Micro-algae biotechnology. Cambridge University Press, Cambridge, pp 27-58

Cheng-Wu Z, Cohen Z, Khozin-Goldberg I, Richmond A (2002) Characterization of growth and arachidonic acid production of Parietochloris incisa comb. Nov. (Trebouxiophyceae, Chlorophyta). J Appl Phycol 14:453-460

Cohen Z (1986) Products from microalgae. In: Richmond A (ed) Handbook for microalgal mass culture. CRC, Boca Raton, pp 421-454

Cohen Z (1990) The production potential of eicosapentaenoic and arachidonic acids by the red alga Porphyridium cruentum. J Am Oil Chem Soc 67:916-920

Cohen Z (1999) Porphyridium cruentum. In: Cohen Z (ed) Chemicals from microalgae. Taylor and Francis, London, pp 1-24

Cohen Z, Reungjitchachawali M, Siangdung W, Tanticharoen M (1993) Production and partial purification of $\gamma$-linolenic acid and some pigments from Spirulina platensis. J Appl Phycol 24:328-312

Erwin JA (1973) Comparative biochemistry of fatty acids in eukaryotic microorganisms. In: Erwin JA (ed) Lipids and biomembranes of eukaryotic microorganisms. Academic, New York, pp 141-143

Falkowski PG, Sukenik A, Herzig R (1989) Nitrogen limitation in lsochrysis galbana (Haptophyceae). 11. Relative abundance of chloroplast proteins. J Phycol 25:471-478

Geider R, La Roche J, Greene R, Olaizola M (1993) Response of the photosynthetic apparatus of Phaeodactylum tricornutum (Bacillariophyceae) to nitrate, phosphate, or iron starvation. J Phycol 29:755-766

Guschina IA, Harwood JL (2006) Lipids and lipid metabolism in eukaryotic algae. Prog Lipid Res 45:160-186

Harwood JL (1998) Membrane lipids in algae. In: Siegenthaler PA, Murata N (eds) Lipids in photosynthesis: structure, function and genetics. Kluwer Academic, Dordrecht, pp 53-64

Henderson RJ, Mackinlay EE (1992) Radiolabelling studies of lipids in the marine cryptomonad Chroomonas salina in relation to fatty acid desaturation. Plant Cell Physiol 33:395-406

Herzig R, Falkowski PG (1989) Nitrogen limitation in Isochrysis galbana (Haptophyceae). I. Photosynthetic energy conversion and growth efficiencies. J Phycol 25:462-471

Hu Q, Zheng Y, Cohen Z, Richmond A (1997) Enhancement of eicosapentaenoic acid and linolenic acid production by manipulating algal density of outdoor cultures of Monodus subterraneus and Spirulina platensis. Eur J Phycol 32:81-86

Hu Q, Sommerfeld M, Jarvis E, Ghirardi M, Posewitz M, Seibert M, Darzins A (2008) Microalgal triacylglycerols as feedstocks for biofuel production: perspectives and advances. Plant J 54:621639

Jiang Y, Chen F, Liang SZ (1999) Production potential of docohexaenoic acid by the heterotrophic marine dinoflagellate Crypthecodinium cohnii. Process Biochem 34:633-637

Khozin-Goldberg I, Cohen Z (2006) The effect of phosphate starvation on the lipid and fatty acid composition of the fresh water eustigmatophyte Monodus subterraneus. Phytochemistry 67:696-701

Khozin-Goldberg I, Bigogno C, Shreshta P, Cohen Z (2002) Nitrogen starvation induces the accumulation of arachidonic acid in the freshwater green alga Parietochloris incisa (Trebouxiophyceae). J Phycol 38:991-994
Kolber Z, Zehr J, Falkowski PG (1988) Effects of growth irradiance and nitrogen limitation on photosynthetic energy conversion in photosystem II. Plant Physiol 88:923-929

Merzlyak MN, Chivkunova OB, Gorelova OA, Reshetnikova IV, Solovchenko AE, Khozin-Goldberg I, Cohen Z (2007) Effect of nitrogen starvation on optical properties, pigments, and arachidonic acid content of the unicellular green alga Parietochloris incisa (Trebouxiophyceae, Chlorophyta). J Phycol 43:833-843

Molina-Grima E, Garcia Camacho F, Acien Fernandez FG (1999) Production of EPA from Phaeodactylum tricornutum. In: Cohen Z (ed) Chemicals from microalgae. Taylor and Francis, London, pp 57-92

Otsuka H (1961) Changes of lipid and carbohydrate contents of Chlorella cells during the sulfur starvation, as studied by the technique of synchronous culture. J Gen Appl Microbiol 7:72-77

Packer A, Li Y, Andersen T, Hu Q, Kuang Y, Sommerfeld M (2011) Growth and neutral lipid synthesis in green microalgae: a mathematical model. Biores Technol 102:111-117

Reitan KI, Rainuzzo JR, Olsen Y (1994) Effect of nutrient limitation on fatty acid and lipid content of marine microalgae. J Phycol 30:972-979

Rhiel E, Krupinska K, Wehrmeyer W (1986) Effects of nitrogen starvation on the function and organization of the photosynthetic membranes in Cryptomonas maculata (Cryptophyceae). Planta 169:361-369

Roessler PG (1990) Environmental control of glycerolipid metabolism in microalgae: commercial implications and future research directions. J Phycol 26:393-399

Sato N, Hagio M, Wada H, Tsuzuki M (2000) Environmental effects on acidic lipids of thylakoid membranes. In: Harwood JL, Quinn PJ (eds) Recent advances in the biochemistry of plant lipids. Portland Press, London, pp 912-914

Seto A, Wang HL, Hesseltine CW (1984) Culture conditions affect eicosapentaenoic acid content of Chlorella minutissima. J Am Oil Chem Soc 61:892-894

Shifrin NS, Chisholm SW (1981) Phytoplankton lipids: interspecific differences and effects of nitrate, silicate and light-dark cycles. J Phycol 17:374-384

Solovchenko AE, Khozin-Goldberg I, Didi-Cohen S, Cohen Z, Merzlyak MN (2008) Effects of light intensity and nitrogen starvation on growth, total fatty acids and arachidonic acid in the green microalga Parietochloris incise. J Appl Phycol 20:245-251

Steinbrenner J, Linden H (2001) Regulation of two carotenoid biosynthesis genes coding for phytoene synthase and carotenoid hydroxylase during stress-induced astaxanthin formation in the green alga Haematococcus pluvialis. Plant Physiol 125:810-817

Thompson GA (1996) Lipids and membrane function in green algae. Biochim Biophys Acta 1302:17-45

Watanabe S, Hirabayashi S, Boussiba S, Cohen Z, Vonshak A, Richmond A (1996) Parietochloris incisa comb. Nov. (Trebuxiophyceae, Chlorophyta). Physiol Res 44:107-108

Wellburn AR (1994) The spectral determination of chlorophyll a and $\mathrm{b}$, as well as total carotenoids, using various solvents with spectrophotometers of different resolutions. J Plant Physiol 144:307-313

Wu ST, Yu ST, Lin LP (2005) Effect of culture conditions on docosahexaenoic acid production by Schizochytrium sp. S31. Process Biochem 40:3103-3108

Yongmanitchai W, Ward OP (1992) Growth and eicosapentaenoic acid production by Phaeodactylum tricurnutum in batch and continuous culture system. J Am Oil Chem Soc 69:584-590 